MATHEMATICS OF COMPUTATION

Volume 77, Number 262, April 2008, Pages 1135-1151

S 0025-5718(07)02082-0

Article electronically published on December 10, 2007

\title{
ON THE POLYNOMIAL REPRESENTATION FOR THE NUMBER OF PARTITIONS WITH FIXED LENGTH
}

\author{
SO RYOUNG PARK, JINSOO BAE, HYUN GU KANG, AND IICKHO SONG
}

\begin{abstract}
In this paper, it is shown that the number $M(n, k)$ of partitions of a nonnegative integer $n$ with $k$ parts can be described by a set of $\tilde{k}$ polynomials of degree $k-1$ in $Q_{\widetilde{k}}$, where $\widetilde{k}$ denotes the least common multiple of the $k$ integers $1,2, \cdots, k$ and $Q_{\widetilde{k}}$ denotes the quotient of $n$ when divided by $\widetilde{k}$. In addition, the sets of the $\widetilde{k}$ polynomials are obtained and shown explicitly for $k=3,4,5$, and 6 .
\end{abstract}

\section{Preliminaries}

Let $N(n)$ and $M(n, k)$ denote the number of partitions of $n$ and that with length $k$ (or with $k$ parts), respectively, where $n$ and $k$ are nonnegative integers. Obviously,

$$
M(n, k)=0, \quad \text { if } k>n .
$$

Defining

$$
N(0)=1
$$

and

$$
M(0,0)=1
$$

for convenience, we have

$$
N(n)=\sum_{k=0}^{n} M(n, k)
$$

While (1.4) allows us to express the number $N(n)$ in terms of $\{M(n, k)\}_{k=0}^{n}$, the number $M(n, k)$ for $k \geq\left\lfloor\frac{n}{3}\right\rfloor$ can be described in terms of $\{N(n)\}_{n=0}^{n-k}$ by

$$
M(n, k)= \begin{cases}N(n-k), & 2 k \geq n \\ & \left(\text { or }\left\lceil\frac{n}{2}\right\rceil \leq k \leq n\right), \\ N(n-k)-\sum_{j=0}^{n-2 k-1} N(j), & 2 k<n \leq 3 k+2 \\ & \left(\text { or }\left\lfloor\frac{n}{3}\right\rfloor \leq k \leq\left\lceil\frac{n}{2}\right\rceil-1\right),\end{cases}
$$

Received by the editor March 9, 2007.

2000 Mathematics Subject Classification. Primary 05A17; Secondary 11P81, 11 P82.

Key words and phrases. Partition, polynomial representation, nonrecursive formula.

This study was supported by the National Research Laboratory (NRL) Program of Korea Science and Engineering Foundation (KOSEF), Ministry of Science and Technology (MOST), under Grant R0A-2005-000-10005-0, for which the authors would like to express their thanks. The authors also wish to express their appreciation of the constructive suggestions and helpful comments from the anonymous reviewers.

(C)2007 American Mathematical Society Reverts to public domain 28 years from publication 
as shown in the Appendix, where $\lceil x\rceil$ and $\lfloor x\rfloor$ denote the smallest integer no smaller than $x$ and the largest integer no larger than $x$, respectively. More generally, it is well known [2, 3, 4, 5, 7] that $M(n, k)$ satisfies the recursion

$$
M(n, k)=M(n-1, k-1)+M(n-k, k) .
$$

Clearly, once $n$ and $k$ are given, we can evaluate $M(n, k)$ recursively with (1.6) using $M(n, 0)=0$ for $n \geq 1$ and $M(0,0)=1$. In this paper we are interested in finding a polynomial representation for the evaluation of $M(n, k)$.

\section{Polynomial Representations}

2.1. A nonrecursive formula. Let us denote by $Q_{n, k}$ and $R_{n, k}$ the quotient and remainder, respectively, of $n$ when divided by $k$. Unless it is ambiguous, we use for brevity the notations $Q_{k}$ and $R_{k}$ for $Q_{n, k}$ and $R_{n, k}$, respectively. Now, from (1.6), we have

$$
\begin{aligned}
M(n, k)-M(n-k, k) & =M(n-1, k-1), \\
M(n-k, k)-M(n-2 k, k) & =M(n-k-1, k-1), \\
& \vdots \\
M\left(k+R_{k}, k\right)-M\left(R_{k}, k\right) & =M\left(k+R_{k}-1, k-1\right),
\end{aligned}
$$

which can be added to produce

$$
M(n, k)=\sum_{i=1}^{\left\lfloor\frac{n}{k}\right\rfloor} M(n+k-k i-1, k-1), \quad k \geq 1
$$

since $R_{k}=0,1, \cdots, k-1$, and consequently, $M\left(R_{k}, k\right)=0$ from (1.1). Based on (2.1), we will show next that $M(n, k)$ can be described by a set of polynomials.

2.2. Simple examples. Let us first obtain polynomial representations for $M(n, k)$ when $k=1,2,3$ using (2.1). Clearly, when $k=1$, we have

$$
\begin{aligned}
M(n, 1) & =\sum_{i=1}^{n} M(n-i, 0) \\
& =1, \quad n \geq 1
\end{aligned}
$$

from (2.1) using $M(0,0)=1$. If we write $n=2 Q_{2}+R_{2}$ when $k=2$, we have $\left\lfloor\frac{n}{2}\right\rfloor=Q_{2}$. Thus, we get

$$
\begin{aligned}
M(n, 2) & =\sum_{i=1}^{Q_{2}} M(n-2 i+1,1) \\
& =Q_{2}
\end{aligned}
$$

from (2.1) using (2.2). Next, writing $n=6 Q_{6}+R_{6}$ when $k=3$, we have $\left\lfloor\frac{n}{3}\right\rfloor=$ $2 Q_{6}+Q_{R_{6}, 3}$. Then, remembering $M\left(R_{6}-1,2\right)=0$ for $R_{6}=0,1,2$ from (1.1), we 
obtain

$$
\begin{aligned}
M(n, 3)= & \sum_{i=1}^{\left\lfloor\frac{n}{3}\right\rfloor} M(n-3 i+2,2) \\
= & \left\{\begin{array}{c}
M(n-1,2)+M(n-4,2)+\cdots+M\left(R_{6}+2,2\right), \\
R_{6}=0,1,2,\left(2 Q_{6} \text { terms added }\right) \\
M(n-1,2)+M(n-4,2)+\cdots+M\left(R_{6}+2,2\right) \\
+M\left(R_{6}-1,2\right), \quad R_{6}=3,4,5,\left(2 Q_{6}+1 \text { terms added }\right)
\end{array}\right. \\
= & \sum_{i=1}^{Q_{6}}\left\{M\left(6(i-1)+R_{6}+2,2\right)+M\left(6(i-1)+R_{6}+5,2\right)\right\}
\end{aligned}
$$

using (2.3) in (2.1), where $\left\{c_{0,3}(r)\right\}_{r=0}^{5}=\{0,0,0,1,1,2\}$. It is interesting to note that $M(n, 3)$ can also be expressed as (4. p. 451)

$$
M(n, 3)=\left\lfloor\frac{n^{2}+6}{12}\right\rfloor .
$$

2.3. The main result. By extending the results (2.2) (2.5) for $M(n, 1), M(n, 2)$, and $M(n, 3)$, we obtain the main result in the form of a theorem below.

Theorem 2.1. When $k \geq 1$, let us write the natural number $n$ as $n=\widetilde{k} Q_{\widetilde{k}}+R_{\widetilde{k}}$, where $\widetilde{k}$ denotes the least common multiple of the $k$ integers $1,2,3, \cdots, k$. Then the number $M(n, k)=M\left(\widetilde{k} Q_{\widetilde{k}}+R_{\widetilde{k}}, k\right)$ of partitions of $n$ with $k$ parts can be specified with the $\widetilde{k}$ polynomials

$$
M\left(\widetilde{k} Q_{\widetilde{k}}+R_{\widetilde{k}}, k\right)=\sum_{i=0}^{k-1} c_{i, k}\left(R_{\widetilde{k}}\right) Q_{\widetilde{k}}^{i}, \quad R_{\widetilde{k}}=0,1, \cdots, \widetilde{k}-1
$$

of degree $k-1$ in $Q_{\widetilde{k}}$. In (2.7), the constant terms $c_{0, k}(r)=M(r, k)$ for $r=$ $0,1, \cdots, \widetilde{k}-1$, are obtained as

$$
c_{0, k}(r)=\sum_{j=1}^{Q_{r, k}} M(r+k-1-j k, k-1),
$$

the coefficients

$$
c_{k-1, k}(r)=\frac{\widetilde{k}^{k-1}}{k !(k-1) !}, \quad k \geq 1
$$


for $r=0,1, \cdots, \widetilde{k}-1$, of the highest degree term are dependent on $k$ but not on $r$, the coefficients

$$
c_{k-2, k}(r)=\frac{\widetilde{k}^{k-2}}{4 k !(k-2) !}\{4 r+k(k-3)\}, \quad k \geq 3
$$

for $r=0,1, \cdots, \tilde{k}-1$, of the second-highest degree term are linear functions of $r$, and the coefficients

$$
c_{1,4}(r)= \begin{cases}\left(r^{2}+2 r\right) / 4, & R_{r, 2}=0 \\ \left(r^{2}+2 r-3\right) / 4, & R_{r, 2}=1\end{cases}
$$

and

$$
c_{k-3, k}(r)=\frac{\widetilde{k}^{k-3}}{288 k !(k-3) !}
$$

$$
\times\left\{144 r^{2}+72 k(k-3) r+k\left(9 k^{3}-58 k^{2}+75 k-2\right)\right\}, \quad k \geq 5
$$

for $r=0,1, \cdots, \widetilde{k}-1$, of the third-highest degree term are quadratic functions of $r$.

Proof. Let us prove the theorem using mathematical induction. Obviously, (2.2)(2.5) imply that (2.7) is true when $k=1,2,3$. Assuming (2.7) is true, let us now show that $M(n, k+1)$ is described by $\widetilde{k+1}$ polynomials of degree $k$ in $Q_{\widetilde{k+1}}$. Putting $n$ as $n=\widetilde{k+1} Q_{\widetilde{k+1}}+R_{\widetilde{k+1}}$, we have $\left\lfloor\frac{n}{k+1}\right\rfloor=\frac{\widetilde{k+1}}{k+1} Q_{\widetilde{k+1}}+Q_{R_{\overparen{k+1}}, k+1}$. Thus, denoting $v_{l}=R_{\widetilde{k+1}}-1+l(k+1)$ for convenience, we get

$$
\begin{aligned}
& M(n, k+1)=\sum_{i=1}^{\left\lfloor\frac{n}{k+1}\right\rfloor} M(n+k-(k+1) i, k)
\end{aligned}
$$

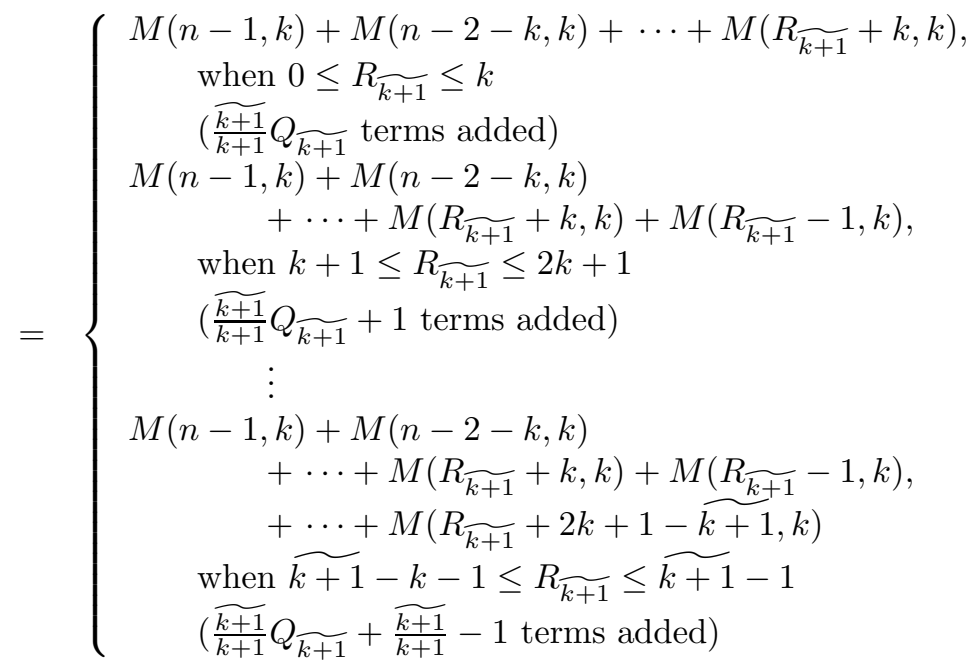


which can be rewritten as

$$
\begin{aligned}
& M(n, k+1)=\sum_{i=1}^{Q_{\widetilde{k+1}}} \sum_{l=1}^{\widetilde{k+1}} M\left(\widetilde{k}\left\{\frac{\widetilde{k+1}}{\widetilde{k}}(i-1)\right\}+R_{\widetilde{k+1}}-1+l(k+1), k\right) \\
& +D\left(R_{\widetilde{k+1}}\right) \\
& =\sum_{i=1}^{Q_{\widetilde{k+1}}} \sum_{l=1}^{\widetilde{k+1}} M\left(\widetilde{k}\left\{\frac{\widetilde{k+1}}{\widetilde{k}}(i-1)+Q_{v_{l}, \widetilde{k}}\right\}+\left(v_{l}-\widetilde{k} Q_{v_{l}, \widetilde{k}}\right), k\right) \\
& +D\left(R_{\widetilde{k+1}}\right) \\
& =\sum_{i=1}^{Q_{\widetilde{k+1}}} \sum_{l=1}^{\widetilde{k+1}}\left[c_{k-1, k}\left(v_{l}-\widetilde{k} Q_{v_{l}, \widetilde{k}}\right)\left\{\widetilde{\frac{k+1}{\widetilde{k}}}(i-1)+Q_{v_{l}, \widetilde{k}}\right\}^{k-1}\right. \\
& +c_{k-2, k}\left(v_{l}-\widetilde{k} Q_{v_{l}, \widetilde{k}}\right)\left\{\frac{\widetilde{k+1}}{\widetilde{k}}(i-1)+Q_{v_{l}, \widetilde{k}}\right\}^{k-2} \\
& +c_{k-3, k}\left(v_{l}-\widetilde{k} Q_{v_{l}, \widetilde{k}}\right)\left\{\frac{\widetilde{k+1}}{\widetilde{k}}(i-1)+Q_{v_{l}, \widetilde{k}}\right\}^{k-3} \\
& \left.+\cdots+c_{0, k}\left(v_{l}-\widetilde{k} Q_{v_{l}, \widetilde{k}}\right)\right]+D\left(R_{\widetilde{k+1}}\right) \\
& =\sum_{i=1}^{Q_{\widetilde{k+1}}}\left\{t_{k-1, k}\left(R_{\widetilde{k+1}}\right)(i-1)^{k-1}+t_{k-2, k}\left(R_{\widetilde{k+1}}\right)(i-1)^{k-2}\right. \\
& \left.+t_{k-3, k}\left(R_{\widetilde{k+1}}\right)(i-1)^{k-3}+\cdots+t_{0, k}\left(R_{\widetilde{k+1}}\right)\right\}+D\left(R_{\widetilde{k+1}}\right)
\end{aligned}
$$

from (2.1) using (2.7) since $0 \leq v_{l}-\widetilde{k} Q_{v_{l}, \widetilde{k}} \leq \widetilde{k}-1$. Here,

$$
D\left(R_{\widetilde{k+1}}\right)=\sum_{j=1}^{Q_{R_{\overparen{k+1}}, k+1}} M\left(R_{\widetilde{k+1}}+k-j(k+1), k\right)
$$

is zero unless $R_{\widetilde{k+1}} \geq k+1$ since $Q_{R_{\overparen{k+1}}, k+1}=0$ when $R_{\widetilde{k+1}} \leq k$,

$$
t_{m, k}\left(R_{\widetilde{k+1}}\right)=\left\{\frac{\widetilde{k+1}}{\widetilde{k}}\right\}^{m} \sum_{l=1}^{\frac{\widehat{k+1}}{k+1}} \bar{c}_{m, k}\left(v_{l}\right), \quad m=0,1, \cdots, k-1
$$

and

$$
\bar{c}_{m, k}(r)=\sum_{t=m}^{k-1}\left(\begin{array}{c}
t \\
t-m
\end{array}\right) c_{t, k}\left(r-\widetilde{k} Q_{r, \widetilde{k}}\right) Q_{r, \widetilde{k}}^{t-m}
$$

Recollecting $R_{\widetilde{k+1}}=0,1, \cdots, \widetilde{k+1}-1$, it is clear from (2.14) that $M(n, k+1)$ is described by $\widetilde{k+1}$ polynomials of degree $k$ in $Q_{\widetilde{k+1}}$, proving that (2.7) holds when 
$k$ is increased by 1 . In addition, we have $c_{0, k+1}\left(R_{\widetilde{k+1}}\right)=D\left(R_{\widetilde{k+1}}\right)$ which is the same as (2.8) when $k+1$ is replaced with $k$. Let us show next that the coefficients of $Q_{\overrightarrow{k+1}}^{k}, Q_{\overrightarrow{k+1}}^{k-1}$, and $Q_{\overrightarrow{k+1}}^{k-2}$ in the polynomial representation of $M(n, k+1)$ are those obtained from (2.9), (2.10), and (2.12), respectively, by replacing $k+1$ for $k$. Concentrating on the terms of $i^{k-1}, i^{k-2}$, and $i^{k-3}$ in (2.14), we have

$$
\begin{aligned}
& M(n, k+1)=\sum_{i=1}^{Q_{\widetilde{k+1}}} t_{k-1, k}\left(R_{\widetilde{k+1}}\right) i^{k-1} \\
& +\sum_{i=1}^{Q_{\widetilde{k+1}}}\left\{(-1)^{0}\left(\begin{array}{c}
k-2 \\
0
\end{array}\right) t_{k-2, k}\left(R_{\widetilde{k+1}}\right)+(-1)^{1}\left(\begin{array}{c}
k-1 \\
1
\end{array}\right) t_{k-1, k}\left(R_{\widetilde{k+1}}\right)\right\} i^{k-2} \\
& +\sum_{i=1}^{Q_{\widetilde{k+1}}}\left\{(-1)^{0}\left(\begin{array}{c}
k-3 \\
0
\end{array}\right) t_{k-3, k}\left(R_{\widetilde{k+1}}\right)+(-1)^{1}\left(\begin{array}{c}
k-2 \\
1
\end{array}\right) t_{k-2, k}\left(R_{\widetilde{k+1}}\right)\right. \\
& \left.+(-1)^{2}\left(\begin{array}{c}
k-1 \\
2
\end{array}\right) t_{k-1, k}\left(R_{\widetilde{k+1}}\right)\right\} i^{k-3}+o\left(i^{k-3}\right) \\
& =t_{k-1, k}\left(R_{\widetilde{k+1}}\right)\left\{H_{1, k-1} Q_{\overparen{k+1}}^{k}+H_{2, k-1} Q_{\overparen{k+1}}^{k-1}+H_{3, k-1} Q_{\overparen{k+1}}^{k-2}+\cdots\right\} \\
& +\left\{t_{k-2, k}\left(R_{\widetilde{k+1}}\right)-(k-1) t_{k-1, k}\left(R_{\widetilde{k+1}}\right)\right\} \\
& \cdot\left\{H_{1, k-2} Q_{\overparen{k+1}}^{k-1}+H_{2, k-2} Q_{\widehat{k+1}}^{k-2}+H_{3, k-2} Q_{\widehat{k+1}}^{k-3}+\cdots\right\} \\
& +\left\{t_{k-3, k}\left(R_{\widetilde{k+1}}\right)-(k-2) t_{k-2, k}\left(R_{\widetilde{k+1}}\right)+\frac{(k-1)(k-2)}{2} t_{k-1, k}\left(R_{\widetilde{k+1}}\right)\right\} \\
& \cdot\left\{H_{1, k-3} Q_{\frac{k-1}{k+1}}^{k-H_{2, k-3}} Q_{\frac{k-3}{k+1}}^{k-H_{3, k-3}} Q_{\frac{k-4}{k+1}}^{k}+\cdots\right\}+o\left(Q_{\frac{k+1}{k+1}}^{k-1}\right. \\
& =H_{1, k-1} t_{k-1, k}\left(R_{\widetilde{k+1}}\right) Q_{\overparen{k+1}}^{k} \\
& +\left[H_{2, k-1} t_{k-1, k}\left(R_{\widetilde{k+1}}\right)+H_{1, k-2}\left\{t_{k-2, k}\left(R_{\widetilde{k+1}}\right)\right.\right. \\
& \left.\left.-(k-1) t_{k-1, k}\left(R_{\widetilde{k+1}}\right)\right\}\right] Q_{\widetilde{k+1}}^{k-1} \\
& +\left[H_{3, k-1} t_{k-1, k}\left(R_{\widetilde{k+1}}\right)+H_{2, k-2}\left\{t_{k-2, k}\left(R_{\widetilde{k+1}}\right)-(k-1) t_{k-1, k}\left(R_{\widetilde{k+1}}\right)\right\}\right. \\
& +H_{1, k-3}\left\{t_{k-3, k}\left(R_{\widetilde{k+1}}\right)-(k-2) t_{k-2, k}\left(R_{\widetilde{k+1}}\right)\right. \\
& \left.\left.+\frac{(k-1)(k-2)}{2} t_{k-1, k}\left(R_{\widetilde{k+1}}\right)\right\}\right] Q_{\overparen{k+1}}^{k-2}+o\left(Q_{\overparen{k+1}}^{k-2}\right) .
\end{aligned}
$$

Here, $H_{j, k}$ denotes the coefficient of (the $j$-th highest degree term) $n^{k+2-j}$ in $\sum_{i=1}^{n} i^{k}$, and can straightforwardly be shown [1] to be $H_{1, k}=\frac{1}{k+1}$ for $k \geq 0$, $H_{2, k}=\frac{1}{2}$ for $k \geq 1$, and $H_{3, k}=\frac{k}{12}$ for $k \geq 2$. Now using (2.9), (2.16), and (2.17), we get 


$$
\begin{aligned}
& t_{k-1, k}\left(R_{\widetilde{k+1}}\right)=\left\{\frac{\widetilde{k+1}}{\widetilde{k}}\right\}^{k-1} \widetilde{\frac{k+1}{k+1}} c_{k-1, k}\left(v_{l}-\widetilde{k} Q_{v_{l}, \widetilde{k}}\right) \\
& = \begin{cases}1, & k-1=0, \\
\left\{\frac{k+1}{\widetilde{k}}\right\}^{k-1} \frac{k+1}{k+1} \frac{\widetilde{\kappa}^{k-1}}{k !(k-1) !}, & k-1 \geq 1,\end{cases} \\
& =\frac{\widetilde{k+1} k}{(k+1) !(k-1) !}, \quad k \geq 1
\end{aligned}
$$

since $c_{0,1}(r)=1$ from (2.2). The result (2.19) can in turn be used in the coefficient of $Q \frac{k}{k+1}$ in (2.18) to produce

$$
\begin{aligned}
c_{k, k+1}\left(R_{\widetilde{k+1}}\right) & =H_{1, k-1} t_{k-1, k}\left(R_{\widetilde{k+1}}\right) \\
& =\frac{1}{k} \frac{\widetilde{k+1}}{(k+1) !(k-1) !} \\
& =\frac{\widetilde{k+1}}{(k+1) ! k !}, \quad k \geq 1 .
\end{aligned}
$$

Next, we have

$$
\begin{aligned}
t_{0,2}\left(R_{6}\right) & =\sum_{l=1}^{2}\left\{c_{0,2}\left(v_{l}-2 Q_{v_{l}, 2}\right)+c_{1,2}\left(v_{l}-2 Q_{v_{l}, 2}\right) Q_{v_{l}, 2}\right\} \\
& =Q_{R_{6}+2,2}+Q_{R_{6}+5,2} \\
& =R_{6}+3
\end{aligned}
$$

after some calculations from (2.16) when $k=2$ since $c_{0,2}(r)=0$ from (2.3) and $c_{1,2}(r)=1$ from (2.20), and

$$
\begin{aligned}
t_{k-2, k}\left(R_{\widetilde{k+1}}\right)= & \left\{\widetilde{\frac{k+1}{\widetilde{k}}}\right\}^{k-2} \sum_{l=1}^{\frac{\overline{k+1}}{k+1}}\left\{c_{k-2, k}\left(v_{l}-\widetilde{k} Q_{v_{l}, \widetilde{k}}\right)\right. \\
& \left.+(k-1) c_{k-1, k}\left(v_{l}-\widetilde{k} Q_{v_{l}, \widetilde{k}}\right) Q_{v_{l}, \widetilde{k}}\right\} \\
= & \left\{\widetilde{\frac{k+1}{\widetilde{k}}}\right\}^{k-2} \sum_{l=1}^{\frac{\widetilde{k+1}}{k+1}}\left\{\frac{\widetilde{k}^{k-2}}{4 k !(k-2) !}\left\{4\left(v_{l}-\widetilde{k} Q_{v_{l}, \widetilde{k}}\right)+k(k-3)\right\}\right. \\
& \left.+(k-1) \frac{\widetilde{k}^{k-1}}{k !(k-1) !} Q_{v_{l}, \widetilde{k}}\right\} \\
= & \left\{\widetilde{\frac{k+1}{\widetilde{k}}}\right\}^{k-2} \frac{\widetilde{k}^{k-2}}{4 k !(k-2) !} \sum_{l=1}^{\frac{k+1}{k+1}}\left\{4 v_{l}+k(k-3)\right\} \\
= & \frac{\widetilde{k+1}}{4 k !(k-2) !}
\end{aligned}
$$




$$
\begin{aligned}
& \times\left[\frac{2}{k+1}\left\{\widetilde{k+1}{ }^{2}+\widetilde{k+1}\left(2 R_{\widetilde{k+1}}+k-1\right)\right\}+\widetilde{\frac{k+1}{k+1}} k(k-3)\right] \\
= & \frac{\widetilde{k+1}}{4(k+1) !(k-2) !}\left\{2 \widetilde{k+1}+4 R_{\widetilde{k+1}}+(k+1)(k-2)\right\}, \quad k \geq 3
\end{aligned}
$$

from (2.16) using (2.10) since

$$
\begin{aligned}
\sum_{l=1}^{\widetilde{k+1}} 4 v_{l} & =4\left\{\left(R_{\widetilde{k+1}}-1\right) \frac{\widetilde{k+1}}{k+1}+\frac{k+1}{2} \frac{\widetilde{k+1}}{k+1}\left(\frac{\widetilde{k+1}}{k+1}+1\right)\right\} \\
& =\frac{2}{k+1}\left\{\widetilde{k+1}^{2}+\widetilde{k+1}\left(2 R_{\widetilde{k+1}}+k-1\right)\right\} .
\end{aligned}
$$

As the result $R_{6}+3$ obtained in (2.21) can also be obtained from (2.22) when we put $k=2$, we note that (2.22) holds for $k \geq 2$. Using (2.19) and (2.22), we have the coefficient of $Q_{\overrightarrow{k+1}}^{k-1}$ in (2.18) as

$$
\begin{aligned}
c_{k-1, k+1}\left(R_{\widetilde{k+1}}\right)= & H_{2, k-1} t_{k-1, k}\left(R_{\widetilde{k+1}}\right) \\
& +H_{1, k-2}\left\{t_{k-2, k}\left(R_{\widetilde{k+1}}\right)-(k-1) t_{k-1, k}\left(R_{\widetilde{k+1}}\right)\right\} \\
= & -\frac{1}{2} t_{k-1, k}\left(R_{\widetilde{k+1}}\right)+\frac{1}{k-1} t_{k-2, k}\left(R_{\widetilde{k+1}}\right) \\
= & -\frac{1}{2} \frac{\widetilde{k+1}}{(k+1) !(k-1) !} \\
& \quad+\frac{\widetilde{k+1}}{4(k+1) !(k-1) !}\left\{2 \widetilde{k+1}+4 R_{\widetilde{k+1}}+(k+1)(k-2)\right\} \\
(2.24) \quad & \frac{\widetilde{k+1}}{4(k+1) !(k-1) !}\left\{4 R_{\widetilde{k+1}}+(k+1)(k-2)\right\}, \quad k \geq 2 .
\end{aligned}
$$

Finally, defining $\alpha_{k}=\frac{\widetilde{k}^{k-3}}{2 k !(k-3) !}, \beta_{k}=\frac{\widetilde{k}^{k-3}}{4(k-1) !(k-4) !}$, and $\gamma_{k}=\frac{\widetilde{k}^{k-3}}{288(k-1) !(k-3) !}\left(9 k^{3}-\right.$ $\left.58 k^{2}+75 k-2\right)$ for convenience, we have 


$$
\begin{aligned}
t_{k-3, k}\left(R_{\widetilde{k+1}}\right)= & \left\{\frac{\widetilde{k+1}}{\widetilde{k}}\right\}^{k-3} \sum_{l=1}^{\widetilde{k+1}}\left\{c_{k-3, k}\left(v_{l}-\widetilde{k} Q_{v_{l}, \widetilde{k}}\right)\right. \\
& +(k-2) c_{k-2, k}\left(v_{l}-\widetilde{k} Q_{v_{l}, \widetilde{k}}\right) Q_{v_{l}, \widetilde{k}} \\
& \left.+\frac{(k-1)(k-2)}{2} c_{k-1, k}\left(v_{l}-\widetilde{k} Q_{v_{l}, \widetilde{k}}\right) Q_{v_{l}, \widetilde{k}}^{2}\right\} \\
= & \left\{\frac{\widetilde{k+1}}{\widetilde{k}}\right\}^{k-3} \sum_{l=1}^{\widetilde{k+1}}\left[\left(v_{l}-\widetilde{k} Q_{v_{l}, \widetilde{k}}\right)^{2} \alpha_{k}+\left(v_{l}-\widetilde{k} Q_{v_{l}, \widetilde{k}}\right) \beta_{k}+\gamma_{k}\right. \\
& +\frac{\widetilde{k}}{4 k !(k-3) !}\left\{4\left(v_{l}-\widetilde{k} Q_{v_{l}, \widetilde{k}}\right)+k(k-3)\right\} Q_{v_{l}, \widetilde{k}} \\
& \left.+\frac{\widetilde{k}}{2 k !(k-1)} Q_{v_{l}, \widetilde{k}}^{2}\right] \\
= & \left\{\frac{\widetilde{k+1}}{\widetilde{k}}\right\}^{k-3} \sum_{l=1}^{\frac{\widetilde{k+1}}{k+1}}\left\{\alpha_{k} v_{l}^{2}+\beta_{k} v_{l}+\gamma_{k}\right\},
\end{aligned}
$$

which can further be shown to be

$$
\begin{aligned}
t_{0,3}\left(R_{12}\right) & =\sum_{l=1}^{3} \sum_{j=0}^{2} c_{j, 3}\left(v_{l}-6 Q_{v_{l}, 6}\right) Q_{v_{l}, 6}^{j} \\
& = \begin{cases}\left(R_{12}^{2}+14 R_{12}+60\right) / 4, & R_{r, 2}=0, \\
\left(R_{12}^{2}+14 R_{12}+57\right) / 4, & R_{r, 2}=1,\end{cases}
\end{aligned}
$$

and

$$
\begin{aligned}
t_{k-3, k}\left(R_{\widetilde{k+1}}\right)= & \left\{\widetilde{\frac{k+1}{\widetilde{k}}}\right\}^{k-3} \frac{\widetilde{k+1}}{6(k+1)}\left[\alpha_{k}(\widetilde{k+1}+k+1)(\widetilde{k+1}+k+1)\right. \\
& +3\left\{2 \alpha_{k}\left(R_{\widetilde{k+1}}-1\right)+\beta_{k}\right\}(\widetilde{k+1}+k+1) \\
& \left.+6\left\{\alpha_{k}\left(R_{\widetilde{k+1}}-1\right)^{2}+\beta_{k}\left(R_{\widetilde{k+1}}-1\right)+\gamma_{k}\right\}\right] \\
= & \left\{\widetilde { \frac { k + 1 } { \widetilde { k } } \} ^ { k - 3 } } \frac { \widetilde { k + 1 } } { 6 ( k + 1 ) } \left[6 \alpha_{k} R_{\widetilde{k+1}}^{2}\right.\right. \\
& +\left\{6 \alpha_{k}(\widetilde{k+1}+k+1)-12 \alpha_{k}+6 \beta_{k}\right\} R_{\widetilde{k+1}} \\
& +\alpha_{k}(\widetilde{k+1}+k+1)(2 \widetilde{k+1}+k+1) \\
& \left.+3(\widetilde{k+1}+k+1)\left(\beta_{k}-2 \alpha_{k}\right)+6\left(\alpha_{k}-\beta_{k}+\gamma_{k}\right)\right] \\
= & \left\{\widetilde{\frac{k+1}{\widetilde{k}}}\right\}^{k-3} \widetilde{\frac{k+1}{(k+1)}}\left[\frac{\widetilde{k}^{k-3}}{2 k !(k-3) !} R_{\widetilde{k+1}}^{2}\right.
\end{aligned}
$$




$$
\begin{aligned}
+ & \left\{\frac{\widetilde{k}^{k-3}}{2 k !(k-3) !}(\widetilde{k+1}+k+1)-\frac{\widetilde{k}^{k-3}}{k !(k-3) !}\right. \\
& \left.+\frac{\widetilde{k}^{k-3}}{4(k-1) !(k-4) !}\right\} R_{\widetilde{k+1}} \\
+ & \frac{\widetilde{k}^{k-3}}{12 k !(k-3) !}(\widetilde{k+1}+k+1)(\widetilde{k+1}+k+1) \\
+ & \frac{1}{2}(\widetilde{k+1}+k+1)\left(\frac{\widetilde{k}^{k-3}}{4(k-1) !(k-4) !}-\frac{\widetilde{k}^{k-3}}{k !(k-3) !}\right) \\
+ & \frac{\widetilde{k}^{k-3}}{2 k !(k-3) !}-\frac{\widetilde{k}^{k-3}}{4(k-1) !(k-4) !} \\
+ & \left.\frac{\widetilde{k}^{k-3}\left(9 k^{3}-58 k^{2}+75 k-2\right)}{288(k-1) !(k-3) !}\right] \\
= & \frac{\widetilde{k+1}_{6(k+1) !(k-3) !}^{k-2}\left[3 R^{2}\right.}{6(k+1}+\frac{3}{2}\{2 \widetilde{k+1}+(k+1)(k-2)\} R_{\widetilde{k+1}} \\
+ & \widetilde{k+1}+\frac{3}{4} \widetilde{k+1}(k+1)(k-2) \\
+ & \left.\frac{9 k^{4}-22 k^{3}-45 k^{2}+10 k+24}{48}\right], \quad k-3 \geq 1,
\end{aligned}
$$

since $\left\{c_{0,3}(r)\right\}_{r=0}^{5}=\{0,0,0,1,1,2\}, c_{1,3}(r)=r$, and $c_{2,3}(r)=3$ from (2.5). The results (2.26) and (2.27), together with (2.19) and (2.22), can now be used in the coefficient of $Q_{\overrightarrow{k+1}}^{k-2}$ in (2.18) to produce

$$
\begin{aligned}
c_{k-2, k+1}\left(R_{\widetilde{k+1}}\right)=\frac{k-1}{12} t_{k-1, k}\left(R_{\widetilde{k+1}}\right)+\frac{1}{2}\left\{t_{k-2, k}\left(R_{\widetilde{k+1}}\right)\right. \\
\left.\quad-(k-1) t_{k-1, k}\left(R_{\widetilde{k+1}}\right)\right\} \\
+\frac{1}{k-2}\left\{t_{k-3, k}\left(R_{\widetilde{k+1}}\right)-(k-2) t_{k-2, k}\left(R_{\widetilde{k+1}}\right)\right. \\
\left.\quad+\frac{(k-1)(k-2)}{2} t_{k-1, k}\left(R_{\widetilde{k+1}}\right)\right\} \\
=\frac{k-1}{12} t_{k-1, k}\left(R_{\widetilde{k+1}}\right)-\frac{1}{2} t_{k-2, k}\left(R_{\widetilde{k+1}}\right)+\frac{1}{k-2} t_{k-3, k}\left(R_{\widetilde{k+1}}\right) \\
=\frac{\widetilde{k+1} k}{12(k+1) !(k-2) !} \\
\quad-\frac{\widetilde{k+1} k-1}{8(k+1) !(k-2) !}\left\{2 \widetilde{k+1}+4 R_{\widetilde{k+1}}+(k+1)(k-2)\right\} \\
\quad+\frac{\widetilde{k+1}}{6(k+1) !(k-2) !}\left[3 R_{\widetilde{k+1}}^{2}+\frac{3}{2}\{2 \widetilde{k+1}+(k+1)(k-2)\} R_{\widetilde{k+1}}\right.
\end{aligned}
$$




$$
\begin{aligned}
& \quad+\widetilde{k+1}^{2}+\frac{3}{4} \widetilde{k+1}(k+1)(k-2) \\
& \left.\quad+\frac{9 k^{4}-22 k^{3}-45 k^{2}+10 k+24}{48}\right] \\
& =\frac{\widetilde{k+1} k-2}{6(k+1) !(k-2) !}\left\{3 R_{\widetilde{k+1}}^{2}+\frac{3}{2}(k+1)(k-2) R_{\widetilde{k+1}}\right. \\
& \left.\quad+\frac{9 k^{4}-22 k^{3}-45 k^{2}+10 k+24}{48}\right\} \\
& =\frac{\widetilde{k+1} k-2}{288(k+1) !(k-2) !}\left[144 R_{\widetilde{k+1}}^{2}+72(k+1)(k-2) R_{\widetilde{k+1}}\right. \\
& \left.\quad+(k+1)\left\{9(k+1)^{3}-58(k+1)^{2}+75(k+1)-2\right\}\right], \quad k \geq 4,
\end{aligned}
$$

and

$$
\begin{aligned}
c_{1,4}\left(R_{12}\right) & =\frac{1}{6} t_{2,3}\left(R_{12}\right)-\frac{1}{2} t_{1,3}\left(R_{12}\right)+t_{0,3}\left(R_{12}\right) \\
& =t_{0,3}\left(R_{12}\right)-3 R_{12}-15 \\
& = \begin{cases}\left(R_{12}^{2}+2 R_{12}\right) / 4, & R_{R_{12}, 2}=0 \\
\left(R_{12}^{2}+2 R_{12}-3\right) / 4, & R_{R_{12}, 2}=1 .\end{cases}
\end{aligned}
$$

The results (2.20), (2.24), (2.28), and (2.29) complete the proof of the theorem.

We would like to mention that, by writing $n=\widetilde{k} q+r$ where $0 \leq q \leq Q_{\widetilde{k}}$, we have

$$
\begin{aligned}
& M(n, k)=\sum_{i=0}^{k-1} c_{i, k}\left(r-\widetilde{k} Q_{r, \widetilde{k}}\right)\left(q+Q_{r, \widetilde{k}}\right)^{i} \\
& =\sum_{i=0}^{k-1}\left\{\left(\begin{array}{l}
i \\
0
\end{array}\right) c_{i, k}\left(r-\widetilde{k} Q_{r, \widetilde{k}}\right)\right. \\
& +\left(\begin{array}{c}
i+1 \\
1
\end{array}\right) c_{i+1, k}\left(r-\widetilde{k} Q_{r, \widetilde{k}}\right) Q_{r, \widetilde{k}}+\left(\begin{array}{c}
i+2 \\
2
\end{array}\right) c_{i+2, k}\left(r-\widetilde{k} Q_{r, \widetilde{k}}\right) Q_{r, \widetilde{k}}^{2} \\
& \left.+\cdots+\left(\begin{array}{c}
k-1 \\
k-i-1
\end{array}\right) c_{k-1, k}\left(r-\widetilde{k} Q_{r, \widetilde{k}}\right) Q_{r, \widetilde{k}}^{k-i-1}\right\} q^{i} \\
& =\sum_{i=0}^{k-1} \bar{c}_{i, k}(r) q^{i}
\end{aligned}
$$

from (2.7) since $Q_{\widetilde{k}}=q+Q_{r, \widetilde{k}}$ and $R_{\widetilde{k}}=r-\widetilde{k} Q_{r, \widetilde{k}}$. The result (2.30) implies that, to compute $M(n, k)$, the $\widetilde{k}$ polynomials in (2.7) can be extended by expressing the number $n$ more generally as the sum $n=\widetilde{k} q+r$ of a multiple of $\widetilde{k}$ and a nonnegative integer, where $q$ does not need to be $Q_{\widetilde{k}}$. For example, when $n=42, M(42,3)=147$ can be evaluated as $M(6 \cdot 7+0,3)=3 \cdot 7^{2}=147, M(6 \cdot 6+6,3)=3 \cdot 6^{2}+6 \cdot 6+3=147$, or $M(6 \cdot 5+12,3)=3 \cdot 5^{2}+12 \cdot 5+12=147, \cdots$ from the polynomials $3 m^{2}$ for $r=0,3 m^{2}+6 m+3$ for $r=6$, and $3 m^{2}+12 m+12$ for $r=12$ (shown in (2.4) and those extended from (2.5) as in (2.30) $) \cdots$. 
2.4. More examples. Let us now obtain the $\widetilde{k}$ polynomials explicitly for $k=$ $4,5,6$. Writing $n=12 Q_{12}+R_{12}$ when $k=4$, we have

$$
M(n, 4)=12 Q_{12}^{3}+3\left(R_{12}+1\right) Q_{12}^{2}+c_{1,4}\left(R_{12}\right) Q_{12}+c_{0,4}\left(R_{12}\right)
$$

after some manipulations, where $\left\{c_{0,4}(r)\right\}_{r=0}^{11}=\{0,0,0,0,1,1,2,3,5,6,9,11\}$. For example, $M(577,4)=M(48 \cdot 12+1,4)=12 \cdot 48^{3}+6 \cdot 48^{2}=1340928$. When $k=5$, we similarly have

$$
\begin{gathered}
M(n, 5)=4500 Q_{60}^{4}+\left(300 R_{60}+750\right) Q_{60}^{3}+\frac{5}{2}\left(3 R_{60}^{2}+15 R_{60}+5\right) Q_{60}^{2} \\
+c_{1,5}\left(R_{60}\right) Q_{60}+c_{0,5}\left(R_{60}\right),
\end{gathered}
$$

where

$$
24 c_{1,5}(r)= \begin{cases}2 r^{3}+15 r^{2}+10 r-60, & R_{r, 2}=0 \\ 2 r^{3}+15 r^{2}+10 r-15, & R_{r, 2}=1\end{cases}
$$

and $\left\{c_{0,5}(r)\right\}_{r=0}^{59}=\{0,0,0,0,0,1,1,2,3,5,7,10, \cdots, 4932\}$. For example, we have $M(577,5)=M(9 \cdot 60+37,5)=4500 \cdot 9^{4}+11850 \cdot 9^{3}+\frac{23335}{2} \cdot 9^{2}+\frac{10183}{2} \cdot 9+831=$ 39154872. Finally, when $k=6$, we can obtain

$$
\begin{aligned}
M(n, 6)= & 9000 Q_{60}^{5}+\left(750 R_{60}+3375\right) Q_{60}^{4}+\frac{25}{3}\left(3 R_{60}^{2}+27 R_{60}+38\right) Q_{60}^{3} \\
& +c_{2,6}\left(R_{60}\right) Q_{60}^{2}+c_{1,6}\left(R_{60}\right) Q_{60}+c_{0,6}\left(R_{60}\right),
\end{aligned}
$$

where

$$
\begin{gathered}
\frac{24}{5} c_{2,6}(r)= \begin{cases}2 r^{3}+27 r^{2}+76 r, & R_{r, 2}=0 \\
2 r^{3}+27 r^{2}+76 r-45, & R_{r, 2}=1,\end{cases} \\
288 c_{1,6}(r)= \begin{cases}r^{4}+18 r^{3}+76 r^{2}+96, & R_{r, 6}=0, \\
r^{4}+18 r^{3}+76 r^{2}-90 r-629, & R_{r, 6}=1,5 \\
r^{4}+18 r^{3}+76 r^{2}-224, & R_{r, 6}=2,4 \\
r^{4}+18 r^{3}+76 r^{2}-90 r-309, & R_{r, 6}=3,\end{cases}
\end{gathered}
$$

and $\left\{c_{0,6}(r)\right\}_{r=0}^{59}=\{0,0,0,0,0,0,1,1,2,3,5,7,11,14, \cdots, 11720\}$. For example, $M(577,6)=M(9 \cdot 60+37,6)=9000 \cdot 9^{5}+31125 \cdot 9^{4}+\frac{128600}{3} \cdot 9^{3}+\frac{58765}{2} \cdot 9^{2}+$ $\frac{60125}{6} \cdot 9+1360=769373455$.

Some of the results are summarized in Tables 1 4. As shown in Table 1, the leading coefficients $c_{k-1, k}(r)$ form a new integer sequence [6] of infinite length.

TABLE 1 . The leading coefficients $\left\{c_{k-1, k}(r)\right\}_{k=1}^{\infty}$.

\begin{tabular}{|c|c|c|c|c|c|c|c|c|c|}
\hline$k$ & 1 & 2 & 3 & 4 & 5 & 6 & 7 & 8 & $\cdots$ \\
\hline \hline$c_{k-1, k}(r)$ & 1 & 1 & 3 & 12 & 4500 & 9000 & 1512630000 & 1452124800000 & $\cdots$ \\
\hline
\end{tabular}


TABLE 2. The coefficients $\left\{c_{j, 4}(r)\right\}_{j=3}^{0}$ for $M(n, 4)=$ $c_{3,4}\left(R_{12}\right) Q_{12}^{3}+c_{2,4}\left(R_{12}\right) Q_{12}^{2}+c_{1,4}\left(R_{12}\right) Q_{12}+c_{0,4}\left(R_{12}\right)$.

\begin{tabular}{|c|c||c|c|}
\hline$r$ & $\left(c_{3,4}(r), c_{2,4}(r), c_{1,4}(r), c_{0,4}(r)\right)$ & $r$ & $\left(c_{3,4}(r), c_{2,4}(r), c_{1,4}(r), c_{0,4}(r)\right)$ \\
\hline 0 & $(12,3,0,0)$ & 1 & $(12,6,0,0)$ \\
2 & $(12,9,2,0)$ & 3 & $(12,12,3,0)$ \\
4 & $(12,15,6,1)$ & 5 & $(12,18,8,1)$ \\
6 & $(12,21,12,2)$ & 7 & $(12,24,15,3)$ \\
8 & $(12,27,20,5)$ & 9 & $(12,30,24,6)$ \\
10 & $(12,33,30,9)$ & 11 & $(12,36,35,11)$ \\
\hline
\end{tabular}

TABLE 3. The coefficients $\left\{c_{j, 5}(r)\right\}_{j=4}^{0}$ for $M(n, 5)=$ $c_{4,5}\left(R_{60}\right) Q_{60}^{4}+c_{3,5}\left(R_{60}\right) Q_{60}^{3}+c_{2,5}\left(R_{60}\right) Q_{60}^{2}+c_{1,5}\left(R_{60}\right) Q_{60}+$ $c_{0,5}\left(R_{60}\right)$.

\begin{tabular}{|c|c||c|c|}
\hline$r$ & $\left(c_{4,5}(r), c_{3,5}(r), c_{2,5}(r), c_{1,5}(r), c_{0,5}(r)\right)$ & $r$ & $\left(c_{4,5}(r), c_{3,5}(r), c_{2,5}(r), c_{1,5}(r), c_{0,5}(r)\right)$ \\
\hline 0 & $(4500,750,25 / 2,-5 / 2,0)$ & 1 & $(4500,1050,115 / 2,1 / 2,0)$ \\
2 & $(4500,1350,235 / 2,3 / 2,0)$ & 3 & $(4500,1650,385 / 2,17 / 2,0)$ \\
4 & $(4500,1950,565 / 2,29 / 2,0)$ & 5 & $(4500,2250,775 / 2,55 / 2,1)$ \\
6 & $(4500,2550,1015 / 2,81 / 2,1)$ & 7 & $(4500,2850,1285 / 2,123 / 2,2)$ \\
8 & $(4500,3150,1585 / 2,167 / 2,3)$ & 9 & $(4500,3450,1915 / 2,229 / 2,5)$ \\
10 & $(4500,3750,2275 / 2,295 / 2,7)$ & 11 & $(4500,4050,2665 / 2,381 / 2,10)$ \\
12 & $(4500,4350,3085 / 2,473 / 2,13)$ & 13 & $(4500,4650,3535 / 2,587 / 2,18)$ \\
14 & $(4500,4950,4015 / 2,709 / 2,23)$ & 15 & $(4500,5250,4525 / 2,855 / 2,30)$ \\
16 & $(4500,5550,5065 / 2,1011 / 2,37)$ & 17 & $(4500,5850,5635 / 2,1193 / 2,47)$ \\
18 & $(4500,6150,6235 / 2,1387 / 2,57)$ & 19 & $(4500,6450,6865 / 2,1609 / 2,70)$ \\
20 & $(4500,6750,7525 / 2,1845 / 2,84)$ & 21 & $(4500,7050,8215 / 2,2111 / 2,101)$ \\
22 & $(4500,7350,8935 / 2,2393 / 2,119)$ & 23 & $(4500,7650,9685 / 2,2707 / 2,141)$ \\
24 & $(4500,7950,10465 / 2,3039 / 2,164)$ & 25 & $(4500,8250,11275 / 2,3405 / 2,192)$ \\
26 & $(4500,8550,12115 / 2,3791 / 2,221)$ & 27 & $(4500,8850,12985 / 2,4213 / 2,255)$ \\
28 & $(4500,9150,13885 / 2,4657 / 2,291)$ & 29 & $(4500,9450,14815 / 2,5139 / 2,333)$ \\
30 & $(4500,9750,15775 / 2,5645 / 2,377)$ & 31 & $(4500,10050,16765 / 2,6191 / 2,427)$ \\
32 & $(4500,10350,17785 / 2,6763 / 2,480)$ & 33 & $(4500,10650,18835 / 2,7377 / 2,540)$ \\
34 & $(4500,10950,19915 / 2,8019 / 2,603)$ & 35 & $(4500,11250,21025 / 2,8705 / 2,674)$ \\
36 & $(4500,11550,22165 / 2,9421 / 2,748)$ & 37 & $(4500,11850,23335 / 2,10183 / 2,831)$ \\
38 & $(4500,12150,24535 / 2,10977 / 2,918)$ & 39 & $(4500,12450,25765 / 2,11819 / 2,1014)$ \\
40 & $(4500,12750,27025 / 2,12695 / 2,1115)$ & 41 & $(4500,13050,28315 / 2,13621 / 2,1226)$ \\
42 & $(4500,13350,29635 / 2,14583 / 2,1342)$ & 43 & $(4500,13650,30985 / 2,15597 / 2,1469)$ \\
44 & $(4500,13950,32365 / 2,16649 / 2,1602)$ & 45 & $(4500,14250,33775 / 2,17755 / 2,1747)$ \\
46 & $(4500,14550,35215 / 2,18901 / 2,1898)$ & 47 & $(4500,14850,36685 / 2,20103 / 2,2062)$ \\
48 & $(4500,15150,38185 / 2,21347 / 2,2233)$ & 49 & $(4500,15450,39715 / 2,22649 / 2,2418)$ \\
50 & $(4500,15750,41275 / 2,23995 / 2,2611)$ & 51 & $(4500,16050,42865 / 2,25401 / 2,2818)$ \\
52 & $(4500,16350,44485 / 2,26853 / 2,3034)$ & 53 & $(4500,16650,46135 / 2,28367 / 2,3266)$ \\
54 & $(4500,16950,47815 / 2,29929 / 2,3507)$ & 55 & $(4500,17250,49525 / 2,31555 / 2,3765)$ \\
56 & $(4500,17550,51265 / 2,33231 / 2,4033)$ & 57 & $(4500,17850,53035 / 2,34973 / 2,4319)$ \\
58 & $(4500,18150,54835 / 2,36767 / 2,4616)$ & 59 & $(4500,18450,56665 / 2,38629 / 2,4932)$ \\
\hline
\end{tabular}


TABLE 4. The coefficients $\left\{c_{j, 6}(r)\right\}_{j=5}^{0}$ for $M(n, 6)=$ $c_{5,6}\left(R_{60}\right) Q_{60}^{5}+c_{4,6}\left(R_{60}\right) Q_{60}^{4}+c_{3,6}\left(R_{60}\right) Q_{60}^{3}+c_{2,6}\left(R_{60}\right) Q_{60}^{2}+$ $c_{1,6}\left(R_{60}\right) Q_{60}+c_{0,6}\left(R_{60}\right)$.

\begin{tabular}{|c|c|c|c|}
\hline$r$ & $\left(c_{5,6}(r), c_{4,6}(r), c_{3,6}(r), c_{2,6}(r), c_{1,6}(r), c_{0,6}(r)\right)$ & $r$ & $\left(c_{5,6}(r), c_{4,6}(r), c_{3,6}(r), c_{2,6}(r), c_{1,6}(r), c_{0,6}(r)\right)$ \\
\hline 0 & $(9000,3375,950 / 3,0,2 / 6,0)$ & 1 & $(9000,4125,1700 / 3,25 / 2,-13 / 6,0)$ \\
\hline 2 & $(9000,4875,2600 / 3,115 / 2,5 / 6,0)$ & 3 & $(9000,5625,3650 / 3,200 / 2,14 / 6,0)$ \\
\hline 4 & $(9000,6375,4850 / 3,360 / 2,50 / 6,0)$ & 5 & $(9000,7125,6200 / 3,525 / 2,77 / 6,0)$ \\
\hline 6 & $(9000,7875,7700 / 3,775 / 2,167 / 6,1)$ & 7 & $(9000,8625,9350 / 3,1040 / 2,230 / 6,1)$ \\
\hline 8 & $(9000,9375,11150 / 3,1400 / 2,374 / 6,2)$ & 9 & $(9000,10125,13100 / 3,1785 / 2,515 / 6,3)$ \\
\hline 10 & $(9000,10875,15200 / 3,2275 / 2,737 / 6,5)$ & 11 & $(9000,11625,17450 / 3,2800 / 2,962 / 6,7)$ \\
\hline 12 & $(9000,12375,19850 / 3,3440 / 2,1310 / 6,11)$ & 13 & $(9000,13125,22400 / 3,4125 / 2,1649 / 6,14)$ \\
\hline 14 & $(9000,13875,25100 / 3,4935 / 2,2135 / 6,20)$ & 15 & $(9000,14625,27950 / 3,5800 / 2,2642 / 6,26)$ \\
\hline 16 & $(9000,15375,30950 / 3,6800 / 2,3302 / 6,35)$ & 17 & $(9000,16125,34100 / 3,7865 / 2,3995 / 6,44)$ \\
\hline 18 & $(9000,16875,37400 / 3,9075 / 2,4889 / 6,58)$ & 19 & $(9000,17625,40850 / 3,10360 / 2,5810 / 6,71)$ \\
\hline 20 & $(9000,18375,44450 / 3,11800 / 2,6962 / 6,90)$ & 21 & $(9000,19125,48200 / 3,13325 / 2,8177 / 6,110)$ \\
\hline 22 & $(9000,19875,52100 / 3,15015 / 2,9635 / 6,136)$ & 23 & $(9000,20625,56150 / 3,16800 / 2,11174 / 6,163)$ \\
\hline 24 & $(9000,21375,60350 / 3,18760 / 2,13010 / 6,199)$ & 25 & $(9000,22125,64700 / 3,20825 / 2,14927 / 6,235)$ \\
\hline 26 & $(9000,22875,69200 / 3,23075 / 2,17177 / 6,282)$ & 27 & $(9000,23625,73850 / 3,25440 / 2,19550 / 6,331)$ \\
\hline 28 & $(9000,24375,78650 / 3,28000 / 2,22274 / 6,391)$ & 29 & $(9000,25125,83600 / 3,30685 / 2,25145 / 6,454)$ \\
\hline 30 & $(9000,25875,88700 / 3,33575 / 2,28427 / 6,532)$ & 31 & $(9000,26625,93950 / 3,36600 / 2,31862 / 6,612)$ \\
\hline 32 & $(9000,27375,99350 / 3,39840 / 2,35750 / 6,709)$ & 33 & $(9000,28125,104900 / 3,43225 / 2,39839 / 6,811)$ \\
\hline 34 & $(9000,28875,110600 / 3,46835 / 2,44405 / 6,931)$ & 35 & $(9000,29625,116450 / 3,50600 / 2,49202 / 6,1057)$ \\
\hline 36 & $(9000,30375,122450 / 3,54600 / 2,54542 / 6,1206)$ & 37 & $(9000,31125,128600 / 3,58765 / 2,60125 / 6,1360)$ \\
\hline 38 & $(9000,31875,134900 / 3,63175 / 2,66299 / 6,1540)$ & 39 & $(9000,32625,141350 / 3,67760 / 2,72770 / 6,1729)$ \\
\hline 40 & $(9000,33375,147950 / 3,72600 / 2,79862 / 6,1945)$ & 41 & $(9000,34125,154700 / 3,77625 / 2,87287 / 6,2172)$ \\
\hline 42 & $(9000,34875,161600 / 3,82915 / 2,95405 / 6,2432)$ & 43 & $(9000,35625,168650 / 3,88400 / 2,103874 / 6,2702)$ \\
\hline 44 & $(9000,36375,175850 / 3,94160 / 2,113090 / 6,3009)$ & 45 & $(9000,37125,183200 / 3,100125 / 2,122717 / 6,3331)$ \\
\hline 46 & $(9000,37875,190700 / 3,106375 / 2,133127 / 6,3692)$ & 47 & $(9000,38625,198350 / 3,112840 / 2,143990 / 6,4070)$ \\
\hline 48 & $(9000,39375,206150 / 3,119600 / 2,155714 / 6,4494)$ & 49 & $(9000,40125,214100 / 3,126585 / 2,167915 / 6,4935)$ \\
\hline 50 & $(9000,40875,222200 / 3,133875 / 2,181037 / 6,5427)$ & 51 & $(9000,41625,230450 / 3,141400 / 2,194702 / 6,5942)$ \\
\hline 52 & $(9000,42375,238850 / 3,149240 / 2,209330 / 6,6510)$ & 53 & $(9000,43125,247400 / 3,157325 / 2,224549 / 6,7104)$ \\
\hline 54 & $(9000,43875,256100 / 3,165735 / 2,240815 / 6,7760)$ & 55 & $(9000,44625,264950 / 3,174400 / 2,257702 / 6,8442)$ \\
\hline 56 & $(9000,45375,273950 / 3,183400 / 2,275702 / 6,9192)$ & 57 & $(9000,46125,283100 / 3,192665 / 2,294395 / 6,9975)$ \\
\hline 58 & $(9000,46875,292400 / 3,202275 / 2,314249 / 6,10829)$ & 59 & $(9000,47625,301850 / 3,212160 / 2,334850 / 6,11720)$ \\
\hline
\end{tabular}

2.5. Remarks. In passing, we would like to make some remarks on several interesting issues.

Remark 1: Applying mathematical induction in (2.1) with the initial result

$$
M(n, k-1)=\frac{n^{k-2}}{(k-1) !(k-2) !}+o\left(n^{k-3}\right)
$$

deduced from (2.2) and (2.3), we can show that

$$
\begin{aligned}
M(n, k) & =\sum_{i=1}^{\left\lfloor\frac{n}{k}\right\rfloor}\left\{\frac{(n+k-k i-1)^{k-2}}{(k-1) !(k-2) !}+o\left(n^{k-3}\right)\right\} \\
& =\frac{1}{(k-1) !(k-2) !} \sum_{i=1}^{\left\lfloor\frac{n}{k}\right\rfloor}\left\{\sum_{j=0}^{k-2}\left(\begin{array}{c}
k-2 \\
j
\end{array}\right)(-k i)^{j} n^{k-2-j}\right\}+o\left(n^{k-2}\right) \\
& =\frac{1}{(k-1) !(k-2) !}\left\{\sum_{j=0}^{k-2}\left(\begin{array}{c}
k-2 \\
j
\end{array}\right)(-k)^{j}\left(\sum_{i=1}^{\left\lfloor\frac{n}{k}\right\rfloor} i^{j}\right) n^{k-2-j}\right\}+o\left(n^{k-2}\right) \\
& =\frac{1}{(k-1) !(k-2) !}\left\{\begin{array}{l}
k-2 \\
j=0
\end{array}\right) \\
(2.38) & =\frac{n^{k-1}}{k !(k-1) !}+o\left(n^{k-2}\right)
\end{aligned}
$$


after some calculations using $\sum_{i=1}^{\left\lfloor\frac{n}{k}\right\rfloor} i^{j}=\frac{n^{j+1}}{(j+1) k^{j+1}}+o\left(n^{j}\right)$ and $\sum_{j=0}^{k-2}\left(\begin{array}{c}k-1 \\ j+1\end{array}\right)(-1)^{j}=$ $\left(\begin{array}{c}k-1 \\ 0\end{array}\right)=1$. This implies that the sum of degrees in $Q_{\widetilde{k}}$ and $R_{\widetilde{k}}$ for any term on the right-hand side of (2.7) is $k-1$ since $n=\widetilde{k} Q_{\widetilde{k}}+R_{\widetilde{k}}$. Consequently, the coefficient $c_{i, k}\left(R_{\widetilde{k}}\right)$ in (2.7) has degree $k-1-i$ in $R_{\widetilde{k}}$ for $i=0,1, \cdots, k-1$.

Remark 2: By replacing $Q_{\widetilde{k}}$ with $\left(n-R_{\widetilde{k}}\right) / \widetilde{k}$ in (2.7), the polynomial in $n$ of (2.38) for the numbers $M(n, k)$ can be obtained explicitly. Specifically, we have

$$
M(n, k)=\frac{n^{k-1}}{k !(k-1) !}+\frac{(k-3) n^{k-2}}{4(k-1) !(k-2) !}+o\left(n^{k-3}\right)
$$

from (2.7) using (2.9) and (2.10). For example, after some straightforward manipulations in (2.4) and (2.31), we have

$$
12 M(n, 3)= \begin{cases}n^{2}, & R_{6}=0 \\ n^{2}-1, & R_{6}=1,5 \\ n^{2}-4, & R_{6}=2,4 \\ n^{2}+3, & R_{6}=3\end{cases}
$$

and

$$
144 M(n, 4)= \begin{cases}n^{3}+3 n^{2}, & R_{12}=0, \\ n^{3}+3 n^{2}-20, & R_{12}=2, \\ n^{3}+3 n^{2}+32, & R_{12}=4, \\ n^{3}+3 n^{2}-36, & R_{12}=6, \\ n^{3}+3 n^{2}+16, & R_{12}=8, \\ n^{3}+3 n^{2}-4, & R_{12}=10, \\ n^{3}+3 n^{2}-9 n+5, & R_{12}=1,7, \\ n^{3}+3 n^{2}-9 n-27, & R_{12}=3,9, \\ n^{3}+3 n^{2}-9 n-11, & R_{12}=5,11 .\end{cases}
$$

Remark 3: As a final remark, let us mention that we have not made use of generating functions in this paper. Recall that, for fixed $k$, the numbers $\{M(n, k)\}_{n=k}^{\infty}$ have the generating function

$$
\begin{aligned}
\sum_{n=k}^{\infty} M(n, k) x^{n-k} & =\frac{1}{(1-x)\left(1-x^{2}\right) \cdots\left(1-x^{k}\right)} \\
& =\frac{1}{\left(1-x^{\tilde{k}}\right)^{k}} \prod_{j=1}^{k} \frac{1-x^{\tilde{k}}}{1-x^{j}}
\end{aligned}
$$

It would be an interesting topic to use this generating function for a possible alternative point of view of the arguments of this paper.

\section{Appendix}

First, decreasing $n$ and $k$ by 1 until $k=1$ in (1.6) and adding all of the resultant equations, we can obtain

$$
M(n, k)=\sum_{j=0}^{k} M(n-k, j)
$$


Since $M(n-k, j)=0$ for $j \geq n-k+1$ from (1.1), we have

$$
\begin{aligned}
M(n, k) & =\sum_{j=0}^{n-k} M(n-k, j)+\sum_{j=n-k+1}^{k} M(n-k, j) \\
& =\sum_{j=0}^{n-k} M(n-k, j) \\
& =N(n-k), \quad 2 k \geq n
\end{aligned}
$$

from (1.4) and (A.1) when $k \geq n-k$. Next, when $k<n-k$, we have

$$
\begin{aligned}
M(n, k) & =\sum_{j=0}^{n-k} M(n-k, j)-\sum_{j=k+1}^{n-k} M(n-k, j) \\
& =N(n-k)-\sum_{j=k+1}^{n-k} M(n-k, j) \\
& =N(n-k)-\sum_{q=0}^{n-2 k-1} M(n-k, q+k+1)
\end{aligned}
$$

from (1.4) and (A.1). Now, if $k+1 \geq(n-k)-(k+1)$, we have $q+k+1 \geq(n-k)-$ $(q+k+1)$ when $q=0,1, \cdots, n-2 k-1$, and consequently, $M(n-k, q+k+1)=$ $N(n-2 k-1-q)$ for $q=0,1, \cdots, n-2 k-1$ from (A.2). In other words, we can rewrite A.3 as

$$
\begin{aligned}
M(n, k) & =N(n-k)-\sum_{q=0}^{n-2 k-1} N(n-2 k-1-q) \\
& =N(n-k)-\sum_{q=0}^{n-2 k-1} N(q), \quad 2 k<n \leq 3 k+2 .
\end{aligned}
$$

\section{REFERENCES}

1. I. S. Gradshteyn and I. M. Ryzhik, Table of Integrals, Series, and Products, New York: Academic, 1980.

2. R. P. Grimaldi, Discrete and Combinatorial Mathematics, Third Ed., Reading: AddisonWesley, 1994.

3. Mathematical Society of Japan, Encyclopedic Dictionary of Mathematics, Second. Ed., Vol. III, Cambridge: MIT Press, 1986.

4. I. Niven, H. S. Zuckerman, and H. L. Montgomery, An Introduction to the Theory of Numbers, Fifth Ed., New York: John Wiley and Sons, 1991. MR1083765 (91i:11001)

5. K. H. Rosen, J. G. Michaels, J. L. Gross, J. W. Grossman, and D. R. Shier, Handbook of Discrete and Combinatorial Mathematics, New York: CRC, 2000. MR1725200(2000g:05001)

6. N. J. A. Sloane and S. Plouffe, Encyclopedia of Integer Sequences, San Diego: Academic, 1995. MR.1327059(96a:11001)

7. R. P. Stanley, Enumerative Combinatorics, Vols. 1 and 2, Cambridge: Cambridge University Press, 1997. MR 1442260 (98a:05001) 
School of Information, Communications, and Electronics Engineering, The Catholic University of Korea, Bucheon 420-743 Korea

E-mail address: srpark@catholic.ac.kr

Department of Information and Communication Engineering, Sejong University, SEOUl 143-747 Korea

E-mail address: baej@sejong.ac.kr

Department of Electrical Engineering and Computer Science, Korea Advanced InStitute of Science and Technology, Daejeon 305-701 Korea

E-mail address: khg@Sejong.kaist.ac.kr

Department of Electrical Engineering and Computer Science, Korea Advanced InStitute of Science and Technology, Daejeon 305-701 Korea

E-mail address: i.song@ieee.org 